Purdue University Purdue e-Pubs

$2-1-2007$

\title{
Gaseous slip flow analysis of a micromachined flow sensor for ultra small flow applications
}

Jaesung Jang

Purdue University

Steven Wereley

Birck Nanotechnology Center, Purdue University, wereley@purdue.edu

Follow this and additional works at: http://docs.lib.purdue.edu/nanodocs

Jang, Jaesung and Wereley, Steven, "Gaseous slip flow analysis of a micromachined flow sensor for ultra small flow applications" (2007). Other Nanotechnology Publications. Paper 51.

http://docs.lib.purdue.edu/nanodocs/51

This document has been made available through Purdue e-Pubs, a service of the Purdue University Libraries. Please contact epubs@purdue.edu for additional information. 


\title{
Gaseous slip flow analysis of a micromachined flow sensor for ultra small flow applications
}

\author{
Jaesung Jang ${ }^{1}$ and Steven T Wereley \\ Microfluidics Laboratory, Birck Nanotechnology Center, School of Mechanical Engineering, \\ Purdue University, West Lafayette, IN 47907, USA \\ E-mail: wereley@purdue.edu
}

Received 24 September 2006, in final form 28 November 2006

Published 22 December 2006

Online at stacks.iop.org/JMM/17/229

\begin{abstract}
The velocity slip of a fluid at a wall is one of the most typical phenomena in microscale gas flows. This paper presents a flow analysis considering the velocity slip in a capacitive micro gas flow sensor based on pressure difference measurements along a microchannel. The tangential momentum accommodation coefficient (TMAC) measurements of a particular channel wall in planar microchannels will be presented while the previous micro gas flow studies have been based on the same TMACs on both walls. The sensors consist of a pair of capacitive pressure sensors, inlet/outlet and a microchannel. The main microchannel is $128.0 \mu \mathrm{m}$ wide, $4.64 \mu \mathrm{m}$ deep and $5680 \mu \mathrm{m}$ long, and operated under nearly atmospheric conditions where the outlet Knudsen number is 0.0137 . The sensor was fabricated using silicon wet etching, ultrasonic drilling, deep reactive ion etching (DRIE) and anodic bonding. The capacitance change of the sensor and the mass flow rate of nitrogen were measured as the inlet-to-outlet pressure ratio was varied from 1.00 to 1.24 . The measured maximum mass flow rate was $3.86 \times 10^{-10} \mathrm{~kg} \mathrm{~s}^{-1}(0.019 \mathrm{sccm})$ at the highest pressure ratio tested. As the pressure difference increased, both the capacitance of the differential pressure sensor and the flow rate through the main microchannel increased. The laminar friction constant $f \cdot R e$, an important consideration in sensor design, varied from the incompressible no-slip case and the mass sensitivity and resolution of this sensor were discussed. Using the current slip flow formulae, a microchannel with much smaller mass flow rates can be designed at the same pressure ratios.
\end{abstract}

\section{Introduction}

Flow rate and pressure distribution are important measurement parameters that can be used to evaluate and control flow systems. Measurements of very small gas flows are especially essential in leak testing, gas chromatography and low-pressure semiconductor manufacturing machines.

\footnotetext{
1 Present address: Laboratory of Integrated Biomedical Micro/ Nanotechnology and Applications, Birck Nanotechnology Center, Bindley Biosciences Center, School of Electrical and Computer Engineering, Purdue University, West Lafayette, IN 47907, USA.
}

To date, a very large number of gas flow sensors using several different principles have been published [1-6]. Most of them can be classified as either thermal-based or pressurebased. Thermal-based flow sensors, which correlate gas flow velocity with cooling of a heated element, can operate as low as $0.1 \mathrm{sccm}\left(1 \mathrm{sccm}=2.083 \times 10^{-8} \mathrm{~kg} \mathrm{~s}^{-1}\right.$ for nitrogen $)$ in the semiconductor industry [7], but mostly they are impractical at sub-sccm flow rates because the gas velocity is too low to generate any significant convective cooling [2, 8]. In fact, most of the micromachined thermal flow sensors measure a flow velocity range of zero to several meters per second 
[1]. In pressure-based flow sensors, the pressure drop has a linear or quadratic relationship with the flow velocity as a fluid passes through restriction such as a microchannel $[2,3]$ or an orifice [4]. Therefore, one or two pressure sensors need to be employed to measure the pressure drops. The pressure drops can be obtained by quantifying a change of capacitance between a fixed electrode and a membrane or a change of resistance on a membrane and these changes are closely related to the flow rate. Generally, capacitive pressure sensors have been appropriate for low flow rates in terms of sensitivity and temperature independence $[2,9,10]$.

Cho et al [2] developed an ultra-sensitive capacitive micro gas flow sensor to measure sub-sccm flow rates. In fact, they demonstrated that a full scale range of $10^{-3} \mathrm{sccm}$ was achieved along with high pressure sensitivities. However, the underlying flow physics of these sensors were not fully presented in their paper and detailed flow analyses considering size effects were not conducted. Further, they made their microchannels by heavy boron doping, which may increase surface roughness. Surface roughness has never been a trivial issue in micro flow analysis.

At small length scales the fundamental nature of gas flows changes [11]. For instance, the no-slip boundary condition at gas-solid interfaces that is commonly used in macroscale (continuum) fluid mechanics is no longer appropriate at small length scales. The Knudsen number $(K n)$ is defined as the ratio of the mean free path of a gas to the characteristic length scale of a flow and it is used to evaluate non-continuum effects. The flow is said to be in the continuum flow regime if $K n<10^{-3}$, while the flow is said to be in the slip flow regime if $10^{-3}<K n<10^{-1}$ [12]. In micro channel gas flows, the characteristic length scale is mostly in the range of microns or tens of microns, while the molecular mean free path is approximately $64 \mathrm{~nm}$ under atmospheric conditions. Consequently, gas flows in microchannels are often in the slip flow regime in which there exists velocity slip at the interfaces. The degree of the velocity slip at the wall can be estimated by the TMAC, which represents the tangential momentum exchange between the flowing gas molecules and the gas-solid boundary. For example, the case of $\sigma=0$ is called specular reflection, meaning zero skin friction and the case of $\sigma=1$ is called diffuse reflection in which the molecules are reflected with zero average tangential velocity [11]. The TMAC depends on the gas type, the solid material, and the surface finish [11] and experimentally observed TMACs range between 0.2 and 1.0 [12].

In this work, we will develop a capacitive micro flow sensor having a straight and uniform planar microchannel that can measure gas flow rates as low as several hundredth $\mathrm{sccm}$. Many micromachined flow sensors have been published so far, but only a few of them reported on this gas flow rate range $[1,2]$. We will also present effects of the velocity slip on friction constants and TMAC measurements of a particular channel wall. There have been many gas flow studies in planar microchannels at atmospheric pressure, but little attention has been paid to a flow analysis considering different TMACs, that is, different velocity slips on the top and bottom walls while the previous micro gas flow measurements have been based on homogeneous TMACs on the entire walls [11-15]. In fact, microchannels are often composed of different materials such as glass and silicon and different walls with different surface finishes. Moreover, the formulae based on the different velocity slips can provide a convenient way to extract the TMAC of particular wall surfaces of microchannels. TMACs of several gases on smooth silicon surfaces were measured using silicon direct bonding [16] where both walls of the microchannels were identical in material and surface finish, that is, uniform TMACs. However, in practice it is not easy to make microchannels whose entire channel walls have the same TMACs with a variety of materials. For example, silicon direct bonding requires highly smooth surfaces for complete bonding, but multiple fabrication steps may increase surface roughness [17]. The TMAC measurements in the current microchannel will be compared with previously reported TMAC values and the derived TMAC expressions can be used in any planar microchannels. These slip flow formulae can be used to design a microchannel element for much smaller gas flow rates in the pressure measurement based flow sensors having capacitors as well as piezoresistors.

\section{Sensor design}

\subsection{Membrane design}

The membrane design of the sensors begins with its thickness, which will be controlled by etching time, so the thickness should be at least $15 \mu \mathrm{m}$ to be practical [17]. Further, it is assumed that the square membrane is isotropic and clamped on the boundary, implying that the deflection and its derivatives are all zero at the membrane boundaries. A nominal membrane thickness of $30 \mu \mathrm{m}$ is chosen as a safer value to avoid etching through the membrane. Another design constraint is that the membrane must resist bursting when exposed to a sudden high pressure difference across the membrane, which can occur when first introducing flow to the sensor. The maximal membrane width that will resist the burst pressure can be computed by

$$
w_{\mathrm{m}} \leqslant t / \sqrt{\frac{5}{16} \frac{p_{\mathrm{f}}}{\tau_{\mathrm{f}}}}
$$

where $w_{\mathrm{m}}, t, p_{\mathrm{f}}$ and $\tau_{\mathrm{f}}$ are the membrane width, the membrane thickness, the fracture pressure and the fracture stress, respectively [18]. For the given width and thickness, the center deflection of the square membrane having negligible residual stress is given by

$$
w_{\mathrm{cnt}}=0.00126 \frac{\Delta p w_{\mathrm{m}}^{4}}{E t^{3}} 12\left(1-v^{2}\right)=k \Delta p
$$

where $w_{\text {cnt }}, E, \Delta p, v$ and $k$ are the membrane deflection at the center, Young's modulus, the pressure difference between the top and the bottom of the membrane, Poisson's ratio, and the proportionality constant, respectively [18]. If the residual stress is dominant and deflections are small compared with the thickness of the membrane, the deflection at the center of the square membrane is given by [19]

$$
w_{\mathrm{cnt}}=0.0821 \frac{\Delta p w_{\mathrm{m}}^{2}}{\sigma_{\mathrm{r}} t}
$$

where $\sigma_{\mathrm{r}}$ is the residual stress. When neither the residual stress nor bending can be neglected, the correction factor $K$ 
was introduced to account for the error in equation (3). The center deflection is then given by [19]

$$
w_{\mathrm{cnt}}=0.0821 \frac{\Delta p w_{\mathrm{m}}^{2}}{\sigma_{\mathrm{r}} t} K \text {. }
$$

According to the small deflection theory, the mid-plane does not begin to stretch significantly until the center deflection of the membrane reaches 0.2-0.4 times the membrane thickness [18]. Further, for ease of manufacture, the capacitor gap is chosen to be equal to the channel height, which needs to be ensured for the desired range of flow rates. The capacitor gap is then chosen such that it satisfies those conditions.

The normalized capacitance change between no flow and flow at a particular set of experimental conditions can be represented as

$$
C^{*}=\frac{\Delta C\left(=C-C_{0}\right)}{C_{0}}=\int_{0}^{1} \int_{0}^{1} \frac{\mathrm{d} \xi \mathrm{d} \zeta}{1-w(\xi, \zeta) / g}-1
$$

where $C_{0}, g, w, \xi$ and $\varsigma$ are the capacitance without membrane deflection, the capacitor gap without membrane deflection, the membrane deflection and lateral dimensionless variables, respectively. The normalized sensitivity, $S$, can be expressed by

$$
S=\frac{1}{C_{0}} \frac{\mathrm{d}(\Delta C)}{\mathrm{d}(\Delta p)}
$$

\subsection{Channel design and slip flow expressions}

When designing the microchannel of this sensor, the following assumptions are made. The flow in the microchannel is steady state, two-dimensional, laminar and isothermal. For relatively low Reynolds number flows in channels with large channel length-to-height ratio, inertial effects in the momentum equations can be neglected [11]. In addition, the flow is not necessarily incompressible because significant pressure drops can occur due to viscous friction in the long microchannel. The assumption of isothermal flow is generally appropriate for low Mach number flows and is particularly accurate in microchannels where the heat generated by viscous friction has an essentially infinite thermal reservoir to flow into $[11,13,20]$.

According to these assumptions, the governing equation for planar microchannels can be expressed as

$$
\frac{\partial p}{\partial z}=\mu \frac{\partial^{2} v}{\partial y^{2}}
$$

where $p, z, \mu, v$ and $y$ are pressure, the streamwise coordinate, dynamic viscosity, streamwise velocity and the depthwise coordinate, respectively. The first-order velocity slip boundary condition on the top and bottom walls under isothermal condition can be written as

$$
\left.v\right|_{y=h}=-\left.\frac{2-\left.\sigma\right|_{y=h}}{\left.\sigma\right|_{y=h}} \lambda\left(\frac{\partial v}{\partial y}\right)\right|_{y=h}=-\left.A \lambda\left(\frac{\partial v}{\partial y}\right)\right|_{y=h}
$$

and

$$
\left.v\right|_{y=-h}=\left.\frac{2-\left.\sigma\right|_{y=-h}}{\left.\sigma\right|_{y=-h}} \lambda\left(\frac{\partial v}{\partial y}\right)\right|_{y=-h}=\left.B \lambda\left(\frac{\partial v}{\partial y}\right)\right|_{y=-h}
$$

where $\lambda, h$ and $\sigma$ are the mean free path of a gas, the channel half height and the TMAC, respectively. The mean free path of a gas is defined as the distance traveled by the molecules between collisions and is given by [11]

$$
\lambda=\frac{1}{\sqrt{2} \pi d^{2} n}
$$

where $n=p /\left(k_{\mathrm{B}} T\right)$ is number density from the ideal gas law, $T$ is temperature, $k_{\mathrm{B}}$ is the Boltzmann constant and $\underline{d}$ is the molecular diameter. The streamwise velocity in these microchannels can be obtained as

$$
\begin{gathered}
v(y, z)=\frac{h^{2}}{2 \mu}\left(-\frac{\mathrm{d} p}{\mathrm{~d} z}\right)\left[1-\left(\frac{y}{h}\right)^{2}+\frac{2(A-B) K n(z)}{[1+(A+B) K n(z)]} \frac{y}{h}\right. \\
\left.+4 B \cdot K n(z)+\frac{2(A-B) K n(z)[1+2 B \cdot K n(z)]}{[1+(A+B) K n(z)]}\right]
\end{gathered}
$$

where $K n(z)$ is the local Knudsen number $(=\lambda(z) /(2 h))[21]$. If the TMACs on the top and bottom walls are the same, $A=$ $B$, equation (11) reduces to

$$
v(y, z)=\frac{h^{2}}{2 \mu}\left(-\frac{\mathrm{d} p}{\mathrm{~d} z}\right)\left[1+4 K n(z) \frac{2-\sigma}{\sigma}-\left(\frac{y}{h}\right)^{2}\right]
$$

which is the same as in [13].

According to mass conservation, the mass flow rate in the microchannels with the different TMACs must be equal to that in the microchannels with the average TMAC. If the difference of the respective pressures of equations (11) and (12) is small at a given streamwise position, the respective volume flow rates must be the same. After integrating and equating both equations, there are an insufficient number of equations to find both $A$ and $B$. However, if the TMAC of one surface is known from another experiment, the TMAC of the remaining surface can be found by

$$
A=\frac{2 C(1+B \cdot K n)-B}{(1-2 C \cdot K n+4 B \cdot K n)}
$$

where $C(=(2-\sigma) / \sigma)$ is computed with the measured average TMAC, and $B$ is known from another experiment [21]. The $K n$ is usually much smaller than 1 in the slip flow regime, so equation (13) can be simplified as

$$
A=2 C-B \text {. }
$$

The mass flow rate can be obtained by applying the ideal gas law and the relationship between pressure and the local Knudsen number at isothermal conditions, $p(z) K n(z)=$ $p_{o} K n_{o}$, and then integrating equation (11) over the cross section. The mass flow rate can be represented by

$$
\begin{aligned}
\dot{m}= & \frac{2 h^{3} w_{\mathrm{c}} p_{\mathrm{o}}^{2}}{3 \mu R_{\mathrm{s}} L T}\left[\Pi^{2}-1+6(A+B) K n_{\mathrm{o}}(\Pi-1)\right. \\
& \left.-6(A-B)^{2} K n_{\mathrm{o}}^{2} \ln \frac{\left(p_{\mathrm{i}}+(A+B) p_{\mathrm{o}} K n_{\mathrm{o}}\right)}{\left(p_{\mathrm{o}}+(A+B) p_{\mathrm{o}} K n_{\mathrm{o}}\right)}\right]
\end{aligned}
$$

where $w_{\mathrm{c}}, R_{\mathrm{s}}, L, \Pi, K n_{\mathrm{o}}$ and $p_{\mathrm{o}}$ are the channel half-width, the specific gas constant, the channel length, the inlet-to-outlet pressure ratio, the Knudsen number at the outlet and the outlet pressure, respectively. Equation (15) can be expressed as

$$
\dot{m}=\frac{2 h^{3} w_{\mathrm{c}} p_{\mathrm{o}}^{2}}{3 \mu R_{\mathrm{s}} L T}\left(\Pi^{2}-1+12 K n_{\mathrm{o}} \frac{2-\sigma}{\sigma}(\Pi-1)\right)
$$

if the TMACs on the top and bottom walls are the same [13-15]. The second-order Knudsen number term in equation (15) is usually much smaller than the first-order Knudsen 
number term, so the TMAC for the remaining side can also be obtained by

$$
A=2 C-B
$$

which is exactly the same as equation (14).

The Darcy-Weisbach formula relates the pressure drop across a known length of a channel or a tube to the flow rate through it for incompressible fully developed flow. According to the Stokes laminar flow theory, the laminar friction constant, $f \cdot R e$, depends on the channel geometry. The friction constant in rectangular channels based on the Stokes theory may be approximated by

$$
\begin{gathered}
(f \cdot R e)_{\mathrm{no}-\mathrm{slip}}=96\left(1-1.3553 a+1.9467 a^{2}\right. \\
\left.-1.7012 a^{3}+0.9564 a^{4}-0.2537 a^{5}\right)
\end{gathered}
$$

where $f, R e$ and $a$ are the friction factor, the Reynolds number based on the hydraulic diameter and the channel height-towidth ratio, respectively [22]. This approximate equation is known to be accurate to within $0.05 \%$ compared to the analytical series solution [22]. Ebert and Sparrow [23] presented numerical data for friction constants in rectangular channels considering velocity slip assuming that the flow is incompressible and fully developed. Their calculations are fit to

$$
\frac{(f \mathrm{Re})_{\mathrm{slip}}}{(f \mathrm{Re})_{\mathrm{no}-\mathrm{slip}}}=\frac{1}{1+\psi \frac{2-\sigma}{\sigma} K n}
$$

where $\psi$ depends on the channel aspect ratio and it is 6.013 for the current microchannel. The friction factor averaged over the channel length for isothermal compressible flow can be expressed as

$$
f=\frac{D_{\mathrm{h}}}{L}\left[\left(f \frac{L_{\mathrm{max}}}{D_{\mathrm{h}}}\right)_{z=0}-\left(f \frac{L_{\mathrm{max}}}{D_{\mathrm{h}}}\right)_{z=L}\right]
$$

where $D_{\mathrm{h}}$ is the hydraulic diameter, and

$$
\left(f \frac{L_{\max }}{D_{\mathrm{h}}}\right)_{z}=\left[\frac{1-\gamma M a^{2}}{\gamma M a^{2}}+\ln \left(\gamma M a^{2}\right)\right]_{z}
$$

where $\gamma$ is the ratio of specific heats and $M a$ is the Mach number [20]. After independent measurements of the friction factor and Reynolds number, the friction constant $f \cdot R e$ is computed.

\section{Fabrication and experimental setup}

The sensors $(8.1 \mathrm{~mm} \times 8.1 \mathrm{~mm})$ consist of a pair of capacitive pressure sensors, one for measuring differential pressure between the inlet and outlet and the other for absolute outlet pressure, and a microchannel to provide flow resistance. Figure 1 shows a schematic view of the sensor. Fluid from the inlet experiences a pressure drop as it flows through the microchannel. The inlet pressure is propagated to the bottom of the differential pressure sensor membrane through the channel on the bottom of the silicon layer. The outlet pressure is transmitted to the top of the differential pressure sensor membrane. The membrane of the differential pressure sensor then deflects in response to the pressure difference between the inlet and outlet of the microchannel.

Figure 2 shows the fabrication procedure for the three layers comprising the sensors-the top glass, middle silicon and bottom glass. The silicon layer starts with 4 inch P-type (100) double side polished $325 \mu \mathrm{m}$ thick silicon wafers $(10-20 \Omega \mathrm{cm})$.

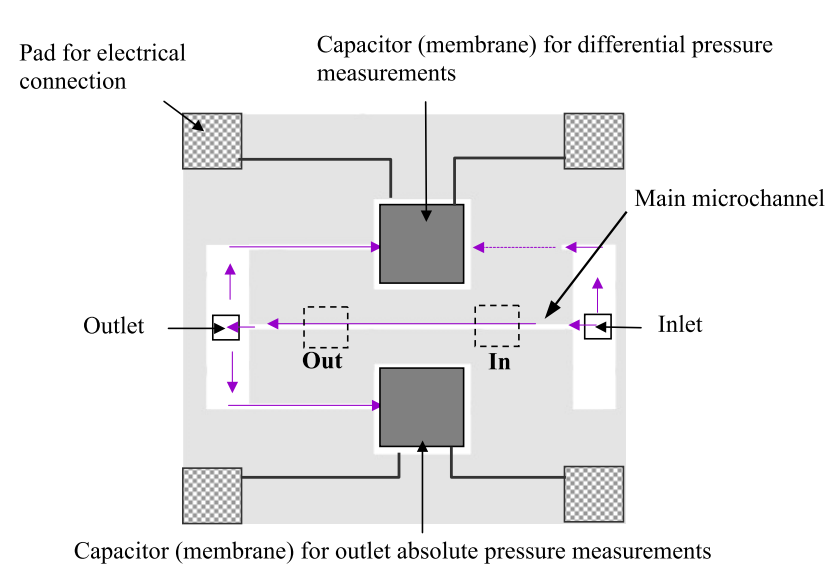

(a)

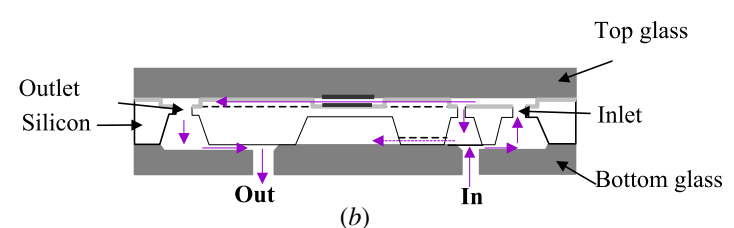

(b)

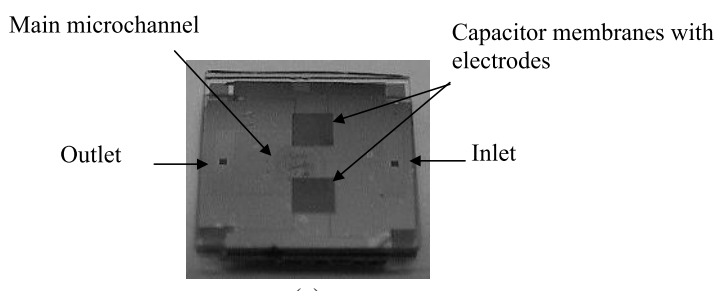

(c)

Figure 1. Schematic of the capacitive micro gas flow sensor $(8.1 \mathrm{~mm} \times 8.1 \mathrm{~mm}),(a)$ top view, $(b)$ side view and $(c)$ picture of the sensor.

(1) The channel on the backside of the silicon layer is made with tetra-methyl-ammonium hydroxide (TMAH) etching to a depth of $6 \mu \mathrm{m}$.

(2) The microchannel and the capacitor gap are etched on the front side of the silicon layer using TMAH at a concentration of $25 \mathrm{wt} \%$ and a temperature of $80^{\circ} \mathrm{C}$. This concentration and temperature are used to obtain smooth surfaces in a controllable period of time [24].

(3) The silicon membranes are made by TMAH etching from the backside with the front side protected with thermallygrown oxide. The etched depth is measured halfway through the process to ensure the proper etch rate. A profilometer (Alpha-step 200) is used to measure etched depths less than $160 \mu \mathrm{m}$ and a high magnification $(50 \times$ objective) optical microscope is used for depths greater than $160 \mu \mathrm{m}$. The membrane thickness is then measured with an optical microscope and it is measured to be $34 \mu \mathrm{m}$ with an accuracy of 3-4 $\mu \mathrm{m}$.

(4) $680 \AA$ thick thermal oxide is grown in a dry atmosphere to serve as an electrical insulation layer. The electrodes on both membranes and feedthrough metals are deposited and patterned on the top of the oxide layer. Gold ( $\mathrm{Au}$ ) (200 $\AA$ ) on chromium $(100 \AA)$ is chosen as the metal, which is known to be resistant to oxidation induced at the glass-silicon interface by the anodic bonding process [25]. 


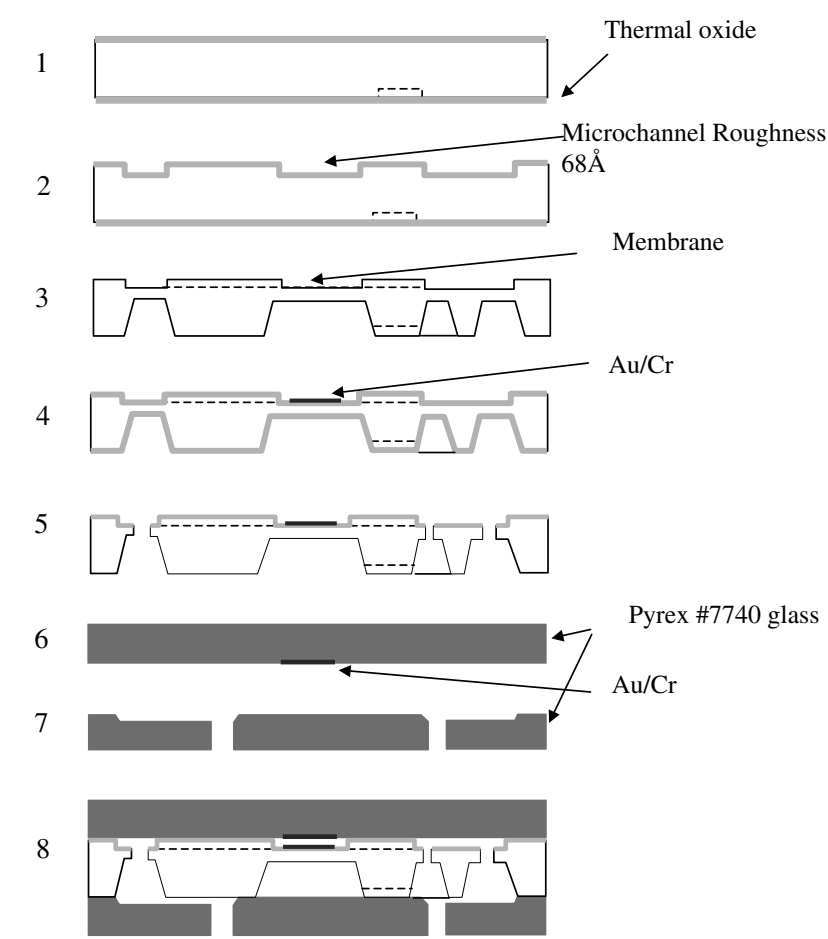

Figure 2. Fabrication procedure of the sensors. 1. Wet etching of a backside channel without a compensation structure. 2. Wet etching of the main microchannels. 3. Wet etching to make membranes. 4. Thermal oxidation for electrical insulation and preventing leak currents and then $\mathrm{Au} / \mathrm{Cr}$ metallization for lower electrodes.

5. DRIE for connecting the frontside and backside of silicon wafer. 6. $\mathrm{Au} / \mathrm{Cr}$ metallization for upper electrodes. 7. Glass etching and ultrasonic drilling. 8. Two anodic bonding.

(5) The flow channel connecting the front side and the backside of the sensor is etched using DRIE.

(6) The electrodes, feedthrough lines and bonding pads are deposited on $0.57 \mathrm{~mm}$ thick corning \#7740 (Pyrex) glass with the same metal composition as used on the silicon layer. Then these patterns are etched with gold and chrome etchants.

(7) Inlet/outlet holes and glass channels are made on another Pyrex glass substrate. Au $(800 \AA) / \mathrm{Cr}(300 \AA)$ is used as a masking layer for glass etching ( $49 \% \mathrm{HF}$ : DI $=1: 1)$ for $16 \mathrm{~min}$ at room temperature. The cross section of the glass channels is 30 times larger than that of the microchannel on the silicon layer, so almost all the pressure drops happen in the microchannels. The glass wafer is then diced and inlet and outlet holes are made using ultrasonic drilling (Bullen Ultrasonics Inc.).

(8) Finally two anodic bonding processes are performed sequentially. First the silicon layer is bonded to the bottom glass and then the top glass chip is bonded to the assembly.

The height and width of the main microchannel are measured with a profilometer (Tencor Alpha-step 200) and an optical microscope (Nikon TE200 40× objective), respectively. The channels are $128.0 \mu \mathrm{m}$ wide, $4.64 \mu \mathrm{m}$ deep and $5680 \mu \mathrm{m}$ long. The two standard deviations $\left(2 \sigma_{\mathrm{s}}\right)$ of the width and depth are $2.25 \%$ and $0.54 \%$ of the measurements, respectively. The surface roughness of the bottom of the channels is also measured with a profilometer and they have

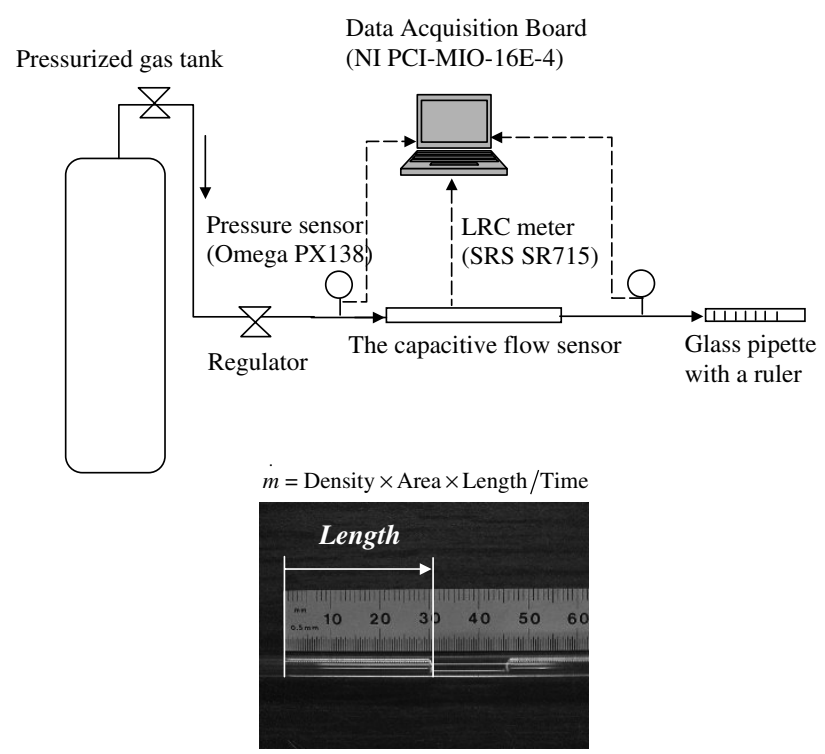

Figure 3. Experimental setup (top) and flow rate measurement scheme (bottom), where the mass flow rate is determined by the distance of a water droplet inside a glass pipette with time and gas density.

an average roughness of $68.0 \AA$ with a standard deviation of $12 \AA$. The Pyrex glass has an average roughness of less than $20 \AA$ according to the manufacturer's datasheet (Sensor Prep Services Inc., USA).

Figure 3 shows the experimental setup and flow rate measurement scheme. In the experiments, dry nitrogen ( $99.995 \%$ pure) is applied to the inlet of the capacitive flow sensor. The mass flow rates are determined by connecting the outlet of the sensor to a $2.4 \mathrm{~mm}$ inner diameter glass pipette containing a water droplet and timing how long it takes the droplet to move a specified distance $[14,15]$. The accuracy of this mass flow measurement scheme strongly depends on the uncertainty in the glass pipette size whose $2 \sigma_{\mathrm{s}}$ is $3.73 \%$ of the measurement. The mass flow rates are measured at steady state as the inlet-to-outlet pressure ratio increases in steps from 1.00 to 1.24 while the outlet pressure is held at the ambient pressure through the droplet mass flow meter. Because of this outlet pressure condition, the outlet absolute pressure sensor is not used in this experiment. The inlet and outlet pressures are obtained with commercial pressure sensors (Omega PX138) via a data acquisition board (NI PCI-MIO-16E-4). These sensors have a typical accuracy of $\pm 0.1 \%$ of the full-scale pressure of $15 \mathrm{psig}$. The inlet and outlet pressure fluctuations measured by these sensors are very small and they are less than $0.04 \%$ of the ambient pressure at steady state. The ambient pressure is measured with a mercury barometer and it is constant during the measurements. The capacitance changes are measured with a high precision LRC meter (Stanford Research Systems SR715). After the pressure readings stabilize, the inlet and outlet pressures and capacitances are measured for at least $8 \mathrm{~min}$.

\section{Results and discussion}

Table 1 summarizes the experimental conditions and physical properties of nitrogen. The hard sphere model has been 


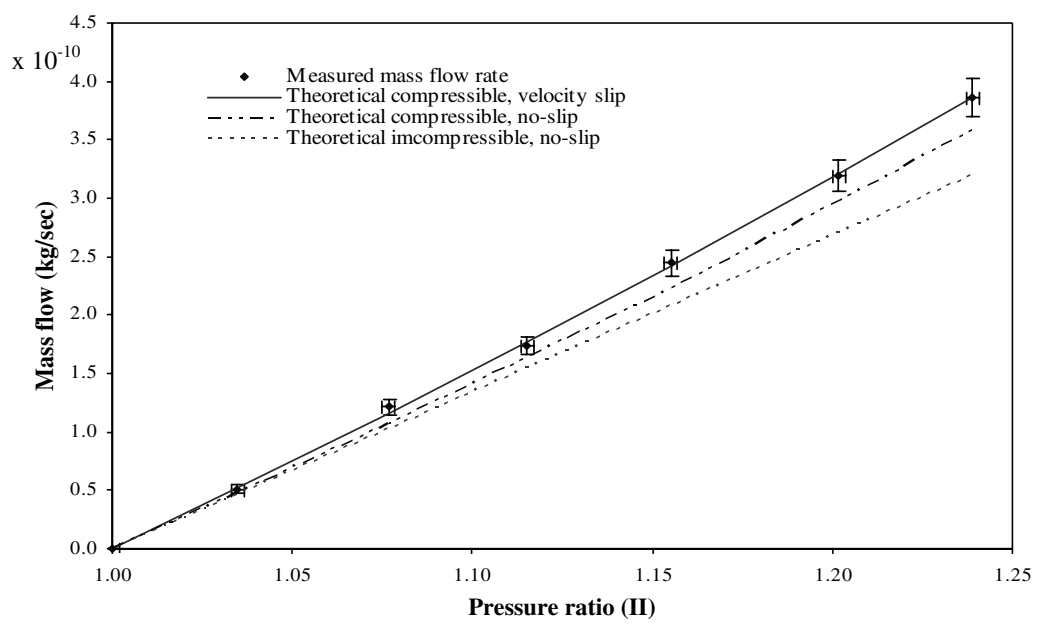

Figure 4. Mass flow rate as a function of the inlet-to-outlet pressure ratio.

Table 1. Experimental conditions and physical properties of nitrogen.

\begin{tabular}{ll}
\hline Parameter & Range or mean values \\
\hline Ambient pressure $\left(p_{\mathrm{a}}\right)$ & $105100 \mathrm{~Pa}$ \\
Inlet-to-outlet pressure ratio $(\Pi)$ & $1.00-1.24$ \\
Mean free path at the outlet $(\lambda)$ & $63.57 \mathrm{~nm}$ \\
Outlet Knudsen number $\left(K n_{\mathrm{o}}\right)$ & 0.0137 \\
Temperature $(T)$ & $295.5 \mathrm{~K}$ \\
Molecular mass & $28.01 \mathrm{~g} \mathrm{~mol}^{-1}$ \\
Reynolds number $(R e)$ & $0.04-0.33$ \\
Ratio of specific heats $(\gamma)$ & 1.4 \\
Molecular diameter & $0.3707 \mathrm{~nm}$ \\
\hline
\end{tabular}

commonly used to calculate the effective molecular diameter and the mean free path of the gas although this model was reported to be slightly different from real gases [26]. Figure 4 shows the measured mass flow rate as a function of the inletto-outlet pressure ratio. The symbols represent the measured mass flow rate and the solid line indicates the analytical expression of equation (16). The maximum measured mass flow rate was $3.86 \times 10^{-10} \mathrm{~kg} \mathrm{~s}^{-1}$ corresponding to a flow rate of $0.019 \mathrm{sccm}$ and an average velocity of $71 \mu \mathrm{m} \mathrm{s}^{-1}$ at the inletto-outlet pressure ratio of 1.24 . The error bars indicate $95 \%$ confidence intervals for both the mass flow rate and the pressure ratio. The relative errors for the mass flow rate and the inlet-to-outlet pressure ratio decreased with the increasing flow rates and they were less than $7.46 \%$ and $4.79 \%$, respectively. The measurements were shown to be in good agreement with the theoretical predictions (equation (16)). The mass flow rate in a rectangular channel with a first-order velocity slip boundary condition was also compared with equation (16) [21] and the relative difference of the mass flow rate is $2.3 \%$ for the current experiments. The pressure drops in the entrance length were negligible compared to the pressure differences over the whole channel length. In fact, the entrance length was calculated as $3.6 \mu \mathrm{m}$ which was $0.06 \%$ of the channel length according to a correlation presented by Chen [27]. It should be pointed out that much smaller gas flow rates can be measured with the current membranes by reducing the size of the microchannel where the slip flow expressions remain effective. For example, a nitrogen flow rate of $9.9 \times 10^{-6}$ sccm is obtained at the same largest pressure difference in $0.64 \mu \mathrm{m}$ high channels, where the outlet Knudsen number is equal to 0.1 (the highest Knudsen number in the slip flow regime), with the same channel aspect ratio.

The dashed line in figure 4 indicates prediction by macro incompressible flow theory. The double dashed line shows the theoretical mass flow rate considering compressibility only, with the outlet Knudsen number set to zero. Obviously, the increased mass flow relative to the incompressible flow case is due to compressibility as well as rarefaction. That can be expected from equations (15) and (16), where the term with the square pressure ratio dependence is associated with compressibility and the other terms containing the outlet Knudsen number are associated with rarefaction. It should be noted that the largest pressure ratio in the experiments was limited by the diameter and length of the homemade droplet flow meter that we used, and the measured pressure ratio range was large enough to observe the slip flow behaviors in these sensors.

The least-squares method was used to obtain the TMAC of nitrogen in the TMAH-etched channel whose sides are made of $680 \AA$ thick thermally-grown silicon dioxide and Pyrex \#7740 glass. The TMAC in this channel was extracted as 0.96 . The extracted TMACs corresponding to the limits of the $95 \%$ confidence intervals of the mass flow rates ranged from 0.74 to 1.36, which was mainly due to the measurement uncertainties of the glass pipette diameter. This value of 0.96 is close to the value of 0.93 obtained in a glass/silicon channel made by DRIE [28]. The TMAC for glass and air combination was reported to be 0.89 [29]. Taking this value for the TMAC of glass and considering that air is mostly composed of nitrogen, the TMAC of nitrogen on the thermal oxide is then calculated as 1.04. This large TMAC can be attributed to the uncertainties of the current mass flow measurements. In fact, if we take the average TMAC of 0.93 for nitrogen in [28] and the TMAC of 0.89 for glass and air combination [29], the TMAC for nitrogen flowing on a silicon surface is obtained as 0.97 . This value is very close to another measurement [11]. Similarly, the TMAC values of gases flowing through any composite and planar channels can be extracted using the equations derived above. 


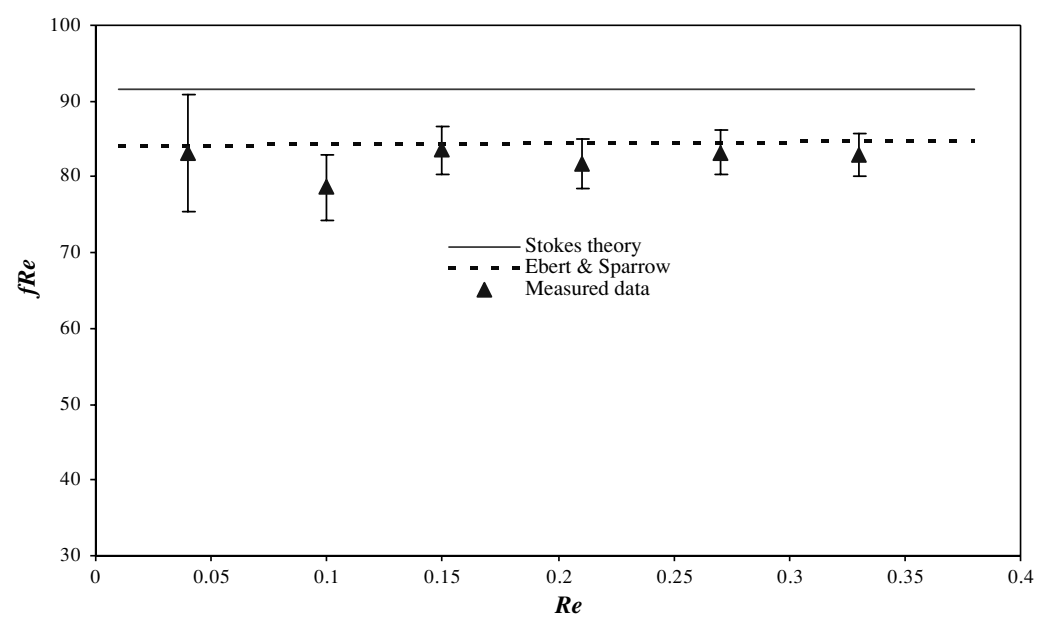

Figure 5. Laminar friction constant as a function of the Reynolds number, where the solid line (Stokes theory) and dashed line (Ebert and Sparrow [23]) indicate 91.52 and 84.44 , respectively.

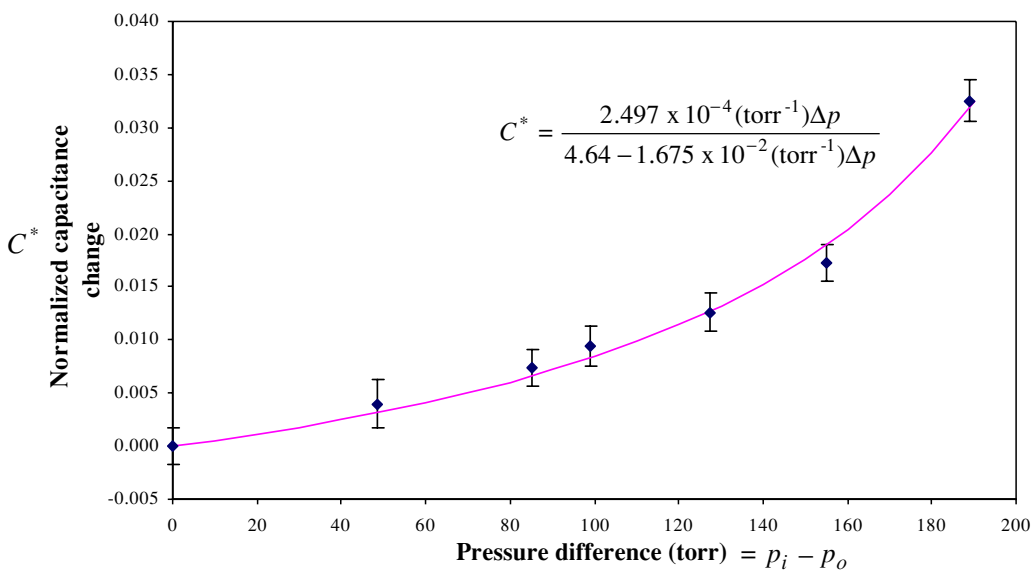

Figure 6. Normalized capacitance change as a function of the pressure difference between the inlet and outlet where the error bars indicate one standard deviation.

Figure 5 shows the friction constant as a function of the Reynolds number based on the hydraulic diameter. The symbols were obtained from flow measurements using equations (20) and (21). The solid line indicates Stokes' law from equation (18). The measured friction constant was 82.18 for nitrogen, which was $10 \%$ lower than the prediction by incompressible no-slip Stokes theory of 91.52. It was observed that these measured friction constants were within the $95 \%$ confidence intervals of 84.43 calculated by Ebert and Sparrow [23] based on the average Knudsen number (equation (19)). The average Knudsen number was used as the representative value over the entire streamwise extent of the channel in this calculation because the Knudsen numbers along the channel were unavailable in this experiment and the Knudsen number changes from entrance to exit are small. It was also observed that the frictional loss becomes lower when velocity slip happens. That is, slip contributes to reducing the pressure difference required to pump at a certain flow rate.

Table 2 shows the material properties of silicon and membrane dimensions. There are uncertainties of these material properties in the table, but the important characteristics such as the membrane deflection and the
Table 2. Material properties of silicon and membrane dimensions.

\begin{tabular}{ll}
\hline Parameter & Range or mean values \\
\hline Young's modulus $(E)$ & $1.7 \times 10^{11} \mathrm{~Pa}[32]$ \\
Poisson's ratio $(v)$ & 0.279 \\
Fracture stress $\left(\tau_{\mathrm{f}}\right)$ & $3 \times 10^{9} \mathrm{~Pa}[32]$ \\
Membrane width $\left(w_{\mathrm{m}}\right)$ & $1700 \mu \mathrm{m}$ \\
Capacitor gap $(g)$ & $4.64 \mu \mathrm{m}$ \\
Proportionality constant $(k)$ & $2.03 \times 10^{-9} \mathrm{~m} \mathrm{Torr}^{-1}$ \\
\hline
\end{tabular}

membrane thickness extracted from the measurements are weakly dependent on the variations of these properties. For example, using two extremely different Poisson's ratios [30], the membrane center deflection changes by only $0.04 \mu \mathrm{m}$ at the highest pressure difference of the current measurements and the extracted membrane thickness changes by $1 \mu \mathrm{m}$. Both of these differences were within the measurement uncertainties.

Figure 6 shows the normalized capacitance change as a function of the pressure difference between the inlet and outlet. The symbols represent the normalized capacitance change measured by the LRC meter. The error bars indicate one standard deviation of more than 700 measurements 


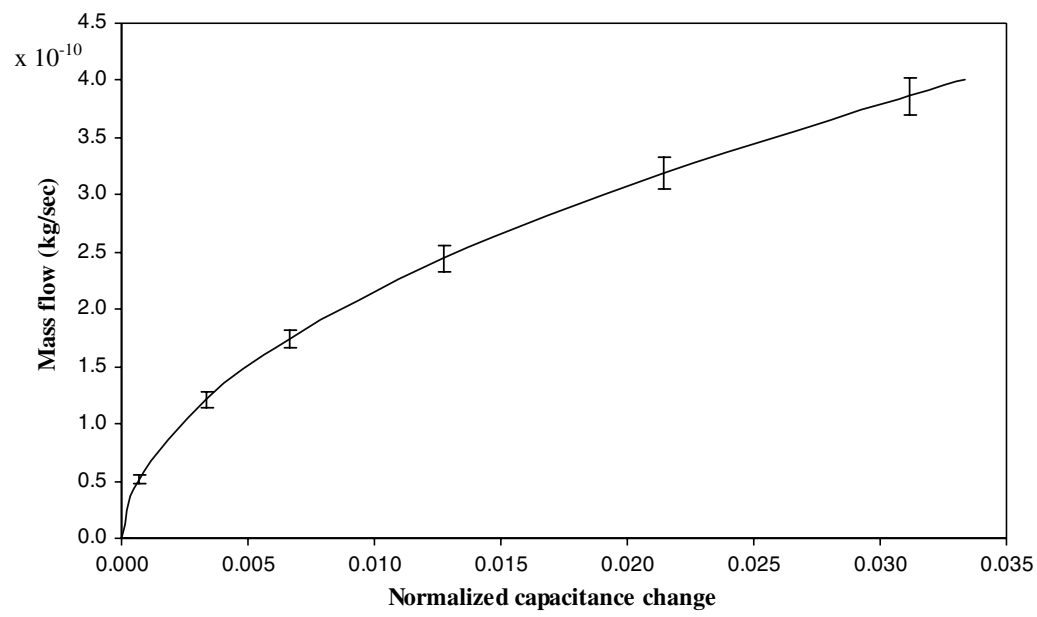

Figure 7. Mass flow rate as a function of the normalized capacitance change and the error bars indicate $95 \%$ confidence intervals.

acquired for each set of operating conditions. As the pressure difference increased, the capacitance change also increased. The normalized sensitivities can be obtained by taking a derivative of the nonlinear fitting curve with respect to the pressure difference. Clearly the sensitivities increased with increasing pressure. At pressure differences up to about 160 Torr, the relationship between the normalized capacitance change and pressure difference was well approximated as linear. The normalized sensitivity in this linear region was obtained as $1.09 \times 10^{-7} \mathrm{mTorr}^{-1}$, which is comparable to $1.36 \times 10^{-7}$ mTorr $^{-1}$ obtained in the linear region of the touch mode capacitive pressure sensor developed by Ko and Wang [31]. The membrane thickness can be estimated using equation (2) and the measurements and it was found to be $36 \mu \mathrm{m}$, which was within the uncertainty of the membrane thickness measured with an optical microscope.

Figure 7 shows the mass flow rates as a function of the normalized capacitance change and represents a calibration curve for the flow sensors, relating the measured capacitance change to the mass flow rate of nitrogen. This graph was obtained from the nonlinear fitting curve in figure 6 (normalized capacitance change versus $\Delta p$ ) and the mass flow rates versus the pressure ratio in figure 4 . The error bars for the mass flow rate indicate the $95 \%$ confidence intervals. As the mass flow rate increased, the normalized capacitance change increased due to the increased pressure difference. The mass flow sensitivity, which was defined as the ratio of a capacitance change to a mass flow change, became larger with the increasing pressure differences, which was consistent with the capacitance versus pressure difference measurements. The minimum detectable mass flow rate due to the capacitance measurement was defined as the smallest mass flow rate corresponding to the capacitance measurement noise and it was obtained as $1.5 \times 10^{-3} \mathrm{sccm}$.

\section{Conclusions}

We presented a slip flow analysis in a capacitive micro gas flow sensor considering velocity slip and compressibility along the microchannels. The sensor consisted of a pair of capacitive pressure sensors, inlet/outlet and a microchannel.
The microchannels were $128.0 \mu \mathrm{m}$ wide, $5680 \mu \mathrm{m}$ long and $4.64 \mu \mathrm{m}$ deep where the outlet Knudsen number was 0.0137. The microchannels had an average roughness of $68.0 \AA$. There was a close agreement between the measurements and theory based on the velocity slip. The maximum measured mass flow rate was $3.86 \times 10^{-10} \mathrm{~kg} \mathrm{~s}^{-1}(0.019 \mathrm{sccm})$ at the inletto-outlet pressure ratio of 1.24. The TMAC of nitrogen in a channel with thermally-grown silicon dioxide and Pyrex \#7740 glass surfaces was obtained as 0.96. Further, if the TMAC of one surface in any planar channel is known from another experiment, the TMAC of the other surface can be found using the formulae derived above. The measured friction constant was within the $95 \%$ confidence intervals of theoretical values based on a compressible flow with velocity slip. Capacitance of the differential pressure sensor increased with the increasing pressure difference between the inlet and outlet. At a pressure difference up to about 160 Torr, the normalized capacitance change was approximately linear with pressure difference and the normalized sensitivity in this region was found to be $1.09 \times$ $10^{-7} \mathrm{mTorr}^{-1}$. The pressure sensitivity of the sensor increased with the increasing pressure differences and the mass flow resolution by the capacitance measurement was obtained as $1.5 \times 10^{-3} \mathrm{sccm}$.

\section{Acknowledgments}

This work was supported by the Indiana 21st Century Research and Technology Fund. The authors would like to thank the staff of the Birck Nanotechnology Center for fabrication support.

\section{References}

[1] Nguyen N T 1997 Micromachined flow sensors—a review Flow Meas. Instrum. 8 7-16

[2] Cho S T, Najafi K, Lowman C E and Wise K D 1992 An ultrasensitive silicon pressure-based microflow sensor IEEE Trans. Electron Devices 39 825-35

[3] Oosterbroek R E, Lammerink T S J, Berenschot J W, Krijnen G J M, Elwenspoek M C and van den Berg A 1999 A micromachined pressure/flow-sensor Sensors Actuators A 77 167-77

[4] Richter M, Wackerle M, Woias P and Hillerich B 1999 A novel flow sensor with high time resolution based on 
differential pressure principle IEEE MEMS 1999 Proc. 1999 IEEE Micro Electro Mechanical Systems (MEMS) pp 118-23

[5] Svedin N, Kälvesten E and Stemme G 2003 A new edge-detected lift force flow sensor J. Microelectromech Syst. 12 344-54

[6] Shin W C and Besser R S 2006 A micromachined thin-film gas flow sensor for microchemical reactors J. Micromech. Microeng. 16 731-41

[7] Tison S A 1996 A critical evaluation of thermal mass flow meters J. Vac. Sci. Technol. A 14 2582-91

[8] Sagi H, Zhao Y and Wereley S T 2004 Wide range flow sensor-vacuum through viscous flow conditions $J$. Vac. Sci. Technol. A 22 1992-9

[9] Puers R 1993 Capacitive sensors: when and how to use them Sensors Actuators A 37-38 93-105

[10] Eaton W P and Smith J H 1997 Micromachined pressure sensors: review and recent developments Smart Mater. Struct. 6 530-9

[11] Karniadakis G E and Beskok A 2002 Micro Flows: Fundamentals and Simulation (New York: Springer)

[12] Gad-el-Hak M 1999 The fluid mechanics of microdevices - the Freemann scholar lecture J. Fluids Eng.-Trans. ASME 121 5-33

[13] Arkilic E B, Schmidt M A and Breuer K S 1997 Gaseous slip flow in long microchannels J. Microelectromech. Syst. 6 167-78

[14] Zohar Y, Lee S Y K, Lee W Y, Jiang L and Tong P 2002 Subsonic gas flow in a straight and uniform microchannel J. Fluid Mech. 472 125-151

[15] Harley J C, Huang Y, Bau H H and Zemel J N 1995 Gas flows in micro-channel J. Fluid Mech. 284 257-74

[16] Arkilic E B, Breuer K S and Schmidt M A 2001 Mass flow and tangential momentum accommodation in silicon micromachined channels J. Fluid Mech. 437 29-43

[17] Madou M 1997 Fundamentals of Microfabrication (Boca Raton, FL: CRC Press)

[18] Timoshenko S P and Woinowsky-Krieger S 1959 Theory of Plates and Shells 2nd edn (New York: McGraw-Hill)
[19] Pedersen M, Meijerink M G H, Olthuis W and Bergveld P 1997 An IC-compatible polyimide pressure sensor with capacitive readout Sensors Actuators A 63 163-8

[20] Fox R W and Mcdonald A T 1998 Introduction to Fluid Mechanics 5th edn (New York: Wiley)

[21] Jang J and Wereley S T 2006 Effective heights and tangential momentum accommodation coefficients of gaseous slip flows in deep reactive ion etching rectangular microchannels J. Micromech. Microeng. 16 493-504

[22] Shah R K and London A L 1978 Laminar Flow Forced Convection in Ducts (Advances in Heat Transfer Series) (New York: Academic)

[23] Ebert W A and Sparrow E M 1965 Slip flow in rectangular and annular ducts J. Basic Eng. 87 1018-24

[24] Shikida M, Masuda T, Uchikawa D and Sato K 2001 Surface roughness of single-crystal silicon etched by TMAH solution Sensors Actuators A 90 223-31

[25] Rogers T and Kowal J 1995 Selection of glass, anodic bonding conditions and material compatibility for silicon-glass capacitive sensors Sensors Actuators A 46-47 113-20

[26] Bird G A 1983 Definition of mean free path for real gases Phys. Fluids 26 3222-3

[27] Chen R-Y 1973 Flow in the entrance region at low Reynolds numbers J. Fluids Eng.-Trans. ASME 95 153-8

[28] Colin S, Lalonde P and Caen R 2004 Validation of a second-order slip flow model in rectangular microchannels Heat Transf. Eng. 25 23-30

[29] Millikan R A 1923 Coefficients of slip in gases and the law of reflection of molecules from the surfaces of solids and liquids Phys. Rev. 21 217-38

[30] Brantley W A 1973 Calculated elastic constants for stress problems associated with semiconductor devices J. Appl. Phys. 44 534-5

[31] Ko W H and Wang Q 1999 Touch mode capacitive pressure sensors Sensors Actuators A 75 242-51

[32] Cho S T, Najafi K and Wise K D 1990 Scaling and dielectrical stress compensation of ultrasensitive boron-doped silicon microstructures Proc. 1990 IEEE Micro Electro Mechanical Systems (MEMS) pp 50-5 